\title{
Mejoría de la sintomatología depresiva en personas mayores gracias al programa universitario de "Las aulas de la experiencia" de la Universidad del País Vasco
}

\author{
Naiara Ozamiz Etxebarria1y Miren Agurtzane Ortiz Jauregi \\ Universidad del País Vasco (España)
}

\begin{abstract}
La esperanza de vida está aumentando en las sociedades del primer mundo por lo que es un objetivo prioritario garantizar una vida satisfactoria y saludable a lo largo de la vida hasta el fin de la misma. Por otro lado, sabemos que la depresión es una de las enfermedades mentales que más prevalece en las personas mayores.

En el presente estudio trabajamos con el alumnado de primer curso de la Universidad del País Vasco para personas mayores, estudiantes todos ellos y ellas de las Aulas de la Experiencia de Bizkaia. Se evaluó la presencia de sintomatología depresiva, así como las relaciones con otras personas con objeto de observar si la asistencia a las aulas está relacionada con una mejoría de la depresión y analizar relaciones entre diferentes variables psicológicas y relacionales. Nuestros resultados sugieren que la Universidad para mayores de 55 años puede mejorar la sintomatología de la depresión y que esta mejoría está relacionada con otras variables psicológicas y relacionales. Subrayamos así la importancia de seguir creando recursos para las personas mayores con el fin de ayudar a prevenir el aislamiento, problemas de salud mental y promover el bienestar de las mismas.
\end{abstract}

Palabras clave: Depresión, envejecimiento saludable, universidad, relaciones sociales.

Improvement of depressive symptomatology in elderly people thanks to the University program of "Classrooms of experience" of the University of the Basque Country. Life expectancy is increasing in first world societies so it is a priority to ensure a satisfactory and healthy life throughout life until the end of it. Furthermore, we know that depression is one of the mental illnesses that most prevails in the elderly. In the actual study, we worked with the students of the first year of the University of the Basque Country, all of them studying in the Experience Classrooms of Biscay. We evaluate the presence of depressive symptomatology, as well as its relationships with other people in order to see if attending to those classes is related with an improvement in depression and to analyze relationships between different psychological and relational variables. Our results suggest that the University for over 55 years old can improve the symptomatology of depression and that this improvement is related to other psychological and relational variables. We underline the importance to continue creating resources for the elderly people in order to help prevent isolation, mental health problems and promote well-being.

Keywords: Depression, healthy aging, university, social relations.

Correspondencia: Naiara Ozamiz Etxebarria. Departamento de Psicoología Evolutiva y de la Educación. Facultad de Educación de la UPV/EHU. Barrio Sarriena s/n. C.P.: 48940. Leioa, Bizkaia (España). E-mail: naiara.ozamiz@ehu.eus 
El aumento de la longevidad es un fenómeno mundial en todas las regiones del mundo, y Europa tiene la mayor proporción de población de 60 años y más.

La esperanza de vida ha aumentado exponencialmente y cada segundo dos personas celebran su 60 cumpleaños en el mundo. Contrariamente a lo que se piensa sobre la etapa de la vejez (Blazer, 2010), hombres y mujeres son más felices con la edad, a pesar de los desafíos que puede traer el envejecimiento (la paradoja de la vejez). Asimismo, y a diferencia de las creencias populares, las cuales reflejan los estereotipos negativos de la edad (Menéndez, Cuevas-Toro, Pérez-Padilla, y Lorence, 2016), las investigaciones sugieren que las habilidades de regulación de las emociones, la estabilidad emocional y el bienestar mejoran con la edad (Jiménez, Izal, y Montorino, 2016). Y, aunque la depresión y la ansiedad son las mayores causas de enfermedad mental en personas mayores (Bryant, Jackson, y Ames, 2008), hay menos trastornos de depresión mayor que en personas más jóvenes (Vivaldi y Barra, 2012). En una investigación realizada en la población del Reino Unido, los resultados sugirieron que, entre todos los grupos de edad, el grupo de personas entre 65 y 79 años tenía los niveles más altos de bienestar personal, y que las personas de más de 90 años tenían niveles más altos de satisfacción y felicidad en comparación con las personas de mediana edad.

Debido al aumento de número de personas mayores en la sociedad, hay muchos más jubilados con mayores oportunidades y opciones de tiempo libre. Diferentes estudios demuestran que para el bienestar de los ancianos es importante que tengan redes sociales y que estén integrados en la sociedad (Oh et al., 2019). Por esta razón, existe una necesidad urgente de crear más recursos para las personas mayores, como una forma de mejorar el envejecimiento saludable y la participación activa e inclusiva en la sociedad.

La depresión, aun siendo el problema más común de la salud mental en los ancianos, todavía está subdiagnosticada y mal tratada (Burroughs et al., 2006), y como tal, en el caso de las personas mayores, que corren un alto riesgo de aislamiento, éste puede elevar el riesgo de depresión. Son frecuentes los tratamientos farmacológicos como tratamiento para la depresión y los trastornos de ansiedad en las personas mayores. En este sentido, son interesantes las iniciativas que promuevan la cohesión y la inclusión social de los jubilados dentro de las comunidades para prevenir problemas de salud. Se ha demostrado que los tratamientos no farmacológicos son eficaces con las personas mayores y mejoran la inclusión y la participación en la sociedad (Laidlaw, Thompson, Gallagher-Thompson, y Dick-Siskin, 2003).

Por otro lado, la jubilación puede ser una época de transición para muchas personas mayores. Considerando la importancia del trabajo en nuestra sociedad, su pérdida puede ser potencialmente estresante para el jubilado (Buendía, 1994). En la jubilación la persona pasa a tener menos actividad y también es una etapa que está relacionada con el inicio de la vejez (Padilla, Góngora, Parra, Díaz, y Manzano, 2016). 
Por lo tanto, el envejecimiento es un proceso donde hay que enfrentar nuevas situaciones y aprender nuevos roles que se puedan desarrollar. Asimismo, también se produce un desglose de las redes sociales, por lo que puede haber una tendencia al aislamiento (Téllez 2004). Estos estresores justifican la necesidad de crear espacios sociales para estas personas

En las últimas décadas, se han desarrollado diversas iniciativas en varios países para preparar a las personas para la jubilación (Bayés, 2012) y abordar cuestiones relacionadas con el ocio, las relaciones familiares y el grado de satisfacción en esta nueva etapa de la vida. Estos programas de preparación para la jubilación tienen resultados muy favorables. Los participantes valoran positivamente estos programas, sintiéndose más tranquilos y obteniendo efectos positivos de estas intervenciones (Midanik, Soghikian, Ransom, y Tekawa, 1995).

Por otro lado, la jubilación puede ser un momento de felicidad y libertad para muchas personas, aunque también hay muchas personas mayores para las que el trabajo ha sido lo más importante de su vida, lo que ha creado su identidad, y por eso pueden aislarse en la jubilación. Los hombres en particular pueden ser especialmente más vulnerables después de jubilarse, ya que pueden reducirse sus redes de apoyo social. Pero la jubilación es más un proceso que un estado y obtener acceso a nuevas y diversas redes sociales puede aumentar la satisfacción con la vida. Por todo ello, es fundamental contar con proyectos que prevengan la soledad y promuevan las relaciones sociales y la actividad mental (Yeung y Zhou, 2017).

Actualmente en la Universidad del País Vasco existen una serie de programas universitarios para personas mayores, las aulas de la experiencia. Varios estudios demuestran que las razones principales de los mayores de 55 años para participar en la educación superior por primera vez como estudiantes en la Universidad son mejorar su cultura, sentirse activos, hacer algo nuevo, mejorar la calidad de vida y conocer gente nueva.

Otros estudios que han investigado el bienestar de los jubilados que acuden a la universidad han demostrado que la participación les hace sentir mucho mejor y más útiles. También hay un sentimiento de orgullo entre los participantes que estudian, y su autoestima se ve reforzada por estas actividades (Sancho, Blasco, Martínez-Mir, y Palmero, 2002).

"Las aulas de la Experiencia" a través de su título universitario en Ciencias Humanas está dirigido principalmente a la población de más de 55 años. Está destinado a todos aquellos que estén interesados en cumplir viejos sueños y completar su formación intelectual. A través de estos estudios, los participantes no sólo cursan diferentes materias de aprendizaje, sino que se benefician también socialización, enriquecimiento personal y la mejora de la salud mental. Es una actividad de cuatro años de duración, 
donde los estudiantes asisten a diferentes asignaturas de humanidades como historia, psicología, artes, política, etc.

Las Aulas de la Experiencia son un proyecto de la Universidad del País Vasco/Euskal Herriko Unibertsitatea y, como tal, participan de su cometido básico: extender la cultura universitaria al conjunto de la Comunidad Autónoma Vasca.

El trabajo realizado por nuestra Universidad en los campos académicos tradicionales (titulaciones propias, maestrías, diplomas, cursos de verano, cátedras de diferentes tipos, etc.) es bien conocido. Sin embargo, resulta más novedosa la actividad desarrollada por las "Aulas de Experiencia" a través de su Grado Universitario en Ciencias Humanas dirigido a la población de más de 55 años.

El objetivo de este estudio es mostrar que "Las aulas de la experiencia" pueden mejorar la sintomatología depresiva de las personas mayores. La hipótesis principal fue que la Universidad para Personas de más de 55 años es una experiencia que mejora la sintomatología depresiva y esto está relacionado con otras variables de la salud mental y relacionales.

\section{MÉTODO}

\section{Participantes}

En este estudio participaron 45 alumnos y alumnas que asistían al primer año de las aulas de la experiencia en la Universidad del País Vasco. Todos los alumnos y alumnas recibieron información sobre el procedimiento de la investigación y aprobaron el consentimiento informado antes de participar en el estudio. Por lo tanto, el procedimiento seguido está aprobado por el Comité de Ética respetando las recomendaciones de la Asociación Médica Mundial, Declaración de Helsinki.

\section{Instrumentos}

La sintomatología depresiva, se midió con el the BSI (Brief Symptom Inventory) (Derogatis y Melisaratos, 1983). El BSI es un cuestionario corto que mide síntomas psicológicos. Es una versión corta del SCL-90-R (Derogatis y Savitz, 2000) y mide nueve escalas de síntomas (somatización, obsesión-compulsión, sensibilidad interpersonal, depresión, ansiedad, hostilidad, ansiedad fóbica, ideación paranoide y psicoticismo.

Las relaciones con los demás se evaluaron utilizando el cuestionario ERA (Echelle de Relations avec les Autres). ERA es un cuestionario de 16 preguntas y mide cuatro dimensiones: apertura a los demás, insight personal y relacional, distancia relacional y gusto por las relaciones.

También se recogieron datos sociodemográficos como el género, edad, estado civil, número de hijos, modo de convivencia y nivel de educación. 


\section{Procedimiento}

Los estudiantes completaron los cuestionarios en clase. La metodología que se utilizó fue test-retest, con ocho meses de diferencia entre los dos tiempos. El test se realizó el primer día del primer curso y el retest el último día del primer curso.

La propia universidad es responsable del diseño, gestión y responsabilidad académica y didáctica.

\section{Análisis estadísticos}

Se realizó el análisis $t$ de Student para observar si había cambios significativos en la sintomatología depresiva desde el principio de curso hasta el final del curso. También se correlaciones para analizar relaciones entre diferentes dimensiones.

\section{RESULTADOS}

Se aplicó el estadístico $t$ de Student para comparar la sintomatología depresiva del test y retest. El valor $p$ de la tabla 1 muestra que hubo una mejoría significativa en la sintomatología de la depresión.

Tabla 1. Mejoría significativa en sintomatología depresiva del BSI

\begin{tabular}{lc}
\hline Dimensión BSI & Análisis estadístico $t$ de Student \\
\hline Depresión & $t=2.3865, p=0.021$ \\
\hline
\end{tabular}

Como se puede ver en la tabla 2, las correlaciones que hicimos entre las diferencias test-retest de las dimensiones de los test BSI y ERA muestran resultados significativos entre la mejoría de las dimensiones somatización y distancia relacional ( $p=0.0366)$; ansiedad y apertura a los demás $(p=0.0010)$; ansiedad fóbica e insight personal y relacional $(p=0.0392)$ e insight personal y relacional y gusto por las relaciones $(p=0.0142)$.

Tabla 2. Correlaciones entre las diferencias test-retest de las dimensiones BSI y ERA

\begin{tabular}{lc}
\hline Somatización y distancia relacional & $r=-0.3126, p=0.0366$ \\
\hline Ansiedad y apertura a los demás & $r=-0.4743, p=0.0010$ \\
\hline Ansiedad fóbica e insight personal y relacional & $r=0.3085, p=0.0392$ \\
\hline
\end{tabular}

La tabla 3 muestra que una mejoría en la depresión está relacionada con la mejoría en la somatización, obsesión compulsión, sensibilidad interpersonal, ansiedad y hostilidad. 
Tabla 3. Correlaciones ente mejorías test-retest del BSI

\begin{tabular}{lccccc}
\hline Sintomatología & Somatización & $\begin{array}{c}\text { Obsesión- } \\
\text { compulsión }\end{array}$ & $\begin{array}{c}\text { Sensibilidad } \\
\text { interpersonal }\end{array}$ & Ansiedad & Hostilidad \\
\hline \multirow{2}{*}{ Depresión } & $r=0.6072$, & $r=0.5888$, & $r=0.6118$, & $r=0.5582$, & $r=0.6613$, \\
& $p=0.0000$ & $p=0.0000$ & $p=0.0000$ & $p=0.0001$ & $p=0.0000$ \\
\hline
\end{tabular}

\section{DISCUSIÓN Y CONCLUSIONES}

Hemos hecho un seguimiento a estudiantes de la Universidad para mayores de 55 años durante el primer año de carrera. Hemos observado que la sintomatología depresiva ha mejorado significativamente. Además, se han obtenido correlaciones positivas entre mejoras psicológicas y variables sobre la relación con los otros. Esto demuestra, que a la vez que hay una mejoría de la sintomatología psicológica, también se mejoran las relaciones con los demás. También se han hallado correlaciones estadísticamente significativas entre la mejoría de la sintomatología depresiva y la mejoría de otra sintomatología psicológica.

Por lo tanto, los resultados sugieren que "las aulas de la experiencia" pueden mejorar la salud la sintomatología depresiva (Yeung y Zhou, 2017). Además, la mejoría sintomatología está relacionada con la mejoría en las relaciones con los demás. Todo esto puede ser resultado de diferentes factores asociados indirectamente con el hecho de participar en las aulas de las experiencias. La asistencia a las clases, aparte de un trabajo cognitivo, suponen una organización del tiempo, autoestima y satisfacción que da sentido a la vida del alumno (Sancho et al., 2002).

Como se ha comentado anteriormente, el aumento de la esperanza de vida es un hecho y el número de personas mayores está creciendo. Todo esto influye en la transformación de la economía y la sociedad del mundo. Por lo tanto, es importante continuar creando recursos para personas mayores ya que cuando se jubilan tienen muy buenas condiciones físicas y psicológicas. Estos recursos previenen el aislamiento y en consecuencia actúan como protectores de la salud mental como pueden ser la ansiedad y la depresión, unas de las mayores causas de enfermedad mental en los adultos mayores (Blazer y Hybels, 2005).

Esta investigación ha demostrado que la universidad para mayores de 55 años puede ser parte de la estrategia para prevenir la enfermedad mental y puede ser una de las iniciativas para mejorar la salud mental y el bienestar de las personas mayores. Por lo que la integración en la sociedad y las redes sociales influyen positivamente en su salud mental (Oh et al., 2019).

También hace frente a los mitos que hay sobre las personas mayores (Menéndez et al., 2016), ya que las personas mayores no carecen de estabilidad emocional y el bienestar (Jiménez et al., 2016). 
Actualmente se recurre con frecuencia a tratamientos farmacológicos para hacer frente a los problemas emocionales de la población general y para las personas mayores en particular. Este estudio ha demostrado que otros recursos como pueden ser la Universidad para Mayores puede mejorar la inclusión y la participación en la sociedad de los adultos, y en consecuencia puede mejorar su salud mental. (Laidlaw et al., 2003).

\section{Agradecimientos}

Nuestro más sincero agradecimiento a los alumnos de las "Aulas de la Experiencia" de la Universidad del País Vasco que han participado en esta investigación. También a los profesores y profesoras de las aulas de la experiencia que nos han facilitado realizar este trabajo.

\section{REFERENCIAS}

Bayés R. (2012). Jubilación activa. Información Psicológica 104, 5-12.

Blazer, D.G. (2010). Protection from late life depression. International Psychogeriatrics, 22(2), 171-173.

Blazer, D.G., y Hybels, C.F. (2005). Origins of depression in later life. Psychological Medicine, 35(9), 1241-1252.

Bryant, C., Jackson, H., y Ames, D. (2008). The prevalence of anxiety in older adults: Methodological issues and a review of the literature. Journal of Affective Disorders, $109(3), 233-250$.

Buendía, J. (1994). Envejecimiento y psicología de la salud. Madrid: Siglo XXI de España Editores.

Burroughs, H., Lovell, K., Morley, M., Baldwin, R., Burns, A., y Chew-Graham, C. (2006). 'Justifiable depression': How primary care professionals and patients view late-life depression? A qualitative study. Family Practice, 23(3), 369-377.

Derogatis, L.R., y Melisaratos, N. (1983). The brief symptom inventory: An introductory report. Psychological Medicine, 13(3), 595-605.

Derogatis, L.R., y Savitz, K.L. (2000). The SCL-90-R and brief symptom inventory (BSI) in primary care. En M.E. Maruish (Ed.), Handbook of psychological assessment in primary care settings (pp. 297-334). Mahwah, NJ: Lawrence Erlbaum Associates Publishers.

Jiménez, G., Izal, M., y Montorio, I. (2016). An intervention program to enhance the wellbeing of the elderly. Pilot study based on positive psychology. Suma Psicológica, 23(1), 51-59.

Laidlaw, K., Thompson, L.W., Gallagher-Thompson, D., y Dick-Siskin, L. (2003). Cognitive behaviour therapy with older people. New York, NY: John Wiley \& Sons Ltd.

Menéndez, S., Cuevas-Toro, A.M, Pérez-Padilla, J., y Lorence, B. (2016). Evaluación de los estereotipos negativos hacia la vejez en jóvenes y adultos. Revista Española de Geriatría y Gerontología, 51(6), 323-328.

Midanik, L.T., Soghikian, K., Ransom, L.J., y Tekawa, I.S. (1995). The effect of retirement on mental health and health behaviors: The kaiser permanente retirement study. The Journals of Gerontology Series B, 50(1), 59-61.

Oh, A., Patel, K., Boscardin, W.J., Max, W., Stephens, C., Ritchie, C.S., y Smith, A.K. (2019). Social Support and Patterns of Institutionalization among Older Adults: A Longitudinal Study. Journal of American Geriatric Society, O(0), 1-6. 
Padilla, D., Góngora, J.M., Parra, A., Díaz, P., y Manzano A. (2016) Personas mayores: innovación y emprendimiento. International Journal of Developmental and Educational Psychology, 1(2), 63-71.

Sancho, C., Blasco, M., Martínez-Mir, R., y Palmero, F. (2002). Análisis de la motivación para el estudio en adultos mayores. REME-Revista Electrónica de Motivación y Emoción, Castellón de la Plana, 5(10), 1-5.

Téllez, V. (2004). Envejecimiento y espacios alternativos de participación social. Emergency, 104, 102-105.

Vivaldi, F., y Barra, E. (2012). Psychological Well-Being, Perceived Social Support and Health Perception Among Older Adults. Terapia Psicológica, 30(2), 23-29.

Yeung D.Y., y Zhou X. (2017). Planning for Retirement: Longitudinal Effect on Retirement Resources and Post-retirement Well-being. Frontiers in Psychology, 7(8), 1300. doi:10.3389/fpsyg.2017.01300

Recibido: 17 de septiembre de 2019 Recepción Modificaciones: 3 de octubre de 2019 Aceptado: 16 de octubre de 2019 